\title{
Neuropsychological Aspects of Prevention and Intervention for FASD: International Perspectives
}

\author{
Christie L. M. Petrenko ${ }^{1}$ Andrew S. Davis ${ }^{2}$
}

Received: 28 February 2017 / Accepted: 28 February 2017 /Published online: 20 March 2017

(C) American Academy of Pediatric Neuropsychology 2017

\begin{abstract}
Although governments around the world are increasingly recognizing the negative impact prenatal alcohol exposure can have on a child's neurodevelopment, the prevalence of fetal alcohol spectrum disorder (FASD) remains highly concerning. Pediatric neuropsychologists have an important role in the prevention of and intervention with children with FASD given the neurocognitive, psychiatric, social, behavioral, and academic problems associated with the condition. This Special Issue reviews different countries' responses to FASD including their prevention and intervention efforts as well as the role that pediatric neuropsychologists have in that country. This introduction discusses the issues that led to the creation of this Special Issue, describes each article, and summarizes common themes raised by contributing countries.
\end{abstract}

Although prenatal exposure to alcohol is a well-known risk factor for the development of physical birth defects and neuropsychological impairment, alcohol-exposed pregnancies continue to occur at a relatively high rate. A recent meta-analysis estimated the global prevalence of alcohol use during pregnancy at 9.8\% (Popova et al. 2017). Regional variation is notable with the highest prevalence in the World Health Organization

Christie L. M. Petrenko

christie.petrenko@rochester.edu

Andrew S. Davis

davis@bsu.edu

1 Mt. Hope Family Center, University of Rochester, 187 Edinburgh St., Rochester, NY 14608, USA

2 Ball State University, Muncie, India
(WHO) European region (25.2\%) and lowest in the Eastern Mediterranean $(0.2 \%)$ and Southeast Asian (1.8\%) regions; rates in the remaining regions ranged from 8.6 to $11.2 \%$. Regional changes in alcohol consumption patterns have also been identified. During the past 5 years, some countries such as China and India have seen an increase in alcohol consumption per capita while the trend in the WHO regions of Africa, Europe, and Americas has been largely stable with some variability per country (WHO 2015). The gender gap in drinking patterns has also been decreasing in some regions, with alcohol consumption increases observed for women, especially for adolescents and young adults (WHO 2005). Different cultural practices and degrees of development with regard to primary, secondary, and tertiary medical and psychological intervention have led to a varied response to alcohol use during pregnancy as well as attitudes about prenatal alcohol exposure.

The term fetal alcohol spectrum disorders (FASD) is an umbrella term used to describe the range of effects on physical dysmorphology, growth, and neurobehavioral functioning that can result from prenatal exposure to alcohol. The diagnostic criteria for the range of conditions that are encapsulated under FASD vary by country, and even within countries, there may be multiple sets of criteria. The prevalence of FASD has been estimated at 2 to $5 \%$ of the population in the USA and other western European countries (May et al. 2009). Higher rates have been found in some regions of the world where heavy binge drinking practices are more prevalent (e.g., May et al. 2013). The financial cost of prenatal alcohol exposure to society is high (Popova et al. 2011). Individuals with FASD have high rates of service utilization across systems (i.e., medical, mental health, special education, child welfare, correctional) and many struggle to live independently and maintain employment as adults (Popova et al. 2011; Streissguth et al. 2004). 


\section{Focus of the Special Issue}

Prenatal alcohol exposure and FASD represent a worldwide public health problem and the prevalence of FASD is estimated at rates equal to or higher than better recognized conditions such as autism spectrum disorders. In finding solutions to prevent and treat FASD, systematic research is needed to inform public policies and best practices. Understanding the approaches being utilized by different countries or regions within their cultural contexts can greatly enhance prevention and intervention efforts. The purpose of this Special Issue is to provide comprehensive reviews of research, policies, and best practices on prevention and intervention in FASD from representative countries around the world to facilitate information sharing and identify future actions to address this major public health problem. This Special Issue also draws attention to the role of pediatric neuropsychologists and psychologists in prevention and intervention in FASD.

Countries were identified for inclusion for this Special Issue based on literature searches of published research on prevention and intervention in FASD. Additional countries with more limited research on FASD were also considered for their unique cultural contexts or active programs or policies relating to FASD. Representation from all regions of the world was sought, although some practical limitations did result in some regions not being reviewed. Submissions for this Special Issue were received from the following countries/ regions: the USA, Canada, Great Britain, South Africa, Poland, China, and Australia. Authors were asked to cover the following topics in their review, when relevant for their country: the epidemiology of alcohol use during pregnancy and FASD; how FASD is conceptualized by governmental, justice, educational, and health systems; research or best practices on prevention and intervention efforts; and identification of future directions and a call to action. Highlights from each article in this Special Issue are briefly presented below, followed by a discussion of themes and future directions identified across countries. One of the strengths of the articles included in this Special Issue is the call to action section which provides a mandate for increased recognition, services, funding, and educational awareness of FASD for their particular region. This introduction concludes with a discussion of how pediatric neuropsychologists and psychologists can contribute to addressing the great prevention and intervention needs for FASD.

\section{Special Issue Article Highlights by Country}

The USA is one of the world's leaders in FASD prevention and intervention research. Their laws have long recognized the potential impact of alcohol on the developing fetus, and since 1989, it has been illegal to sell alcohol without a warning label regarding these effects. There are several governmental agencies providing research funding and leadership in attempting to prevent and reduce alcohol use by pregnant woman as well as provide tertiary interventions for children and their families. In the first article in this Special Issue, Davis, Hoover, Moore, and Petrenko (this issue) provide an overview of these activities and discuss the role that pediatric neuropsychologists can play in increasing both research and service provision to children and families affected by FASD. Despite the relatively high level of funding and number of services relative to many other countries and regions of the world, Davis et al. point out there are a number of areas where improvements are needed. Many people affected by FASD cannot access FASD diagnostic and intervention services, which is in part due to limitations in a knowledgeable and skilled workforce (e.g., health care providers, educators). Davis et al. advocate for increased involvement, engagement, and funding in governmental, health care, educational, and juvenile justice systems for working with children with FASD and to prevent prenatal alcohol exposure.

Along with the USA, Canada dedicates the most resources towards addressing FASD prevention and intervention. In the second article in this Special Issue, Pei, Tremblay, McNeil, Poole, and McFarlane (this issue) review Canadian research and clinical efforts and illustrate the role that nationwide research plays in integrating research and practice. Similar to what is found in the USA, despite widespread prevention efforts, alcohol-exposed pregnancies remain relatively high. From a clinical perspective, Pei et al. describe the newly released diagnostic guidelines as outlined by Cook et al. (2016; as cited by Pei et al., this issue). Given that diagnostic guidelines are still evolving, pediatric neuropsychologists are encouraged to review other country's guidelines as this will help readers understand how FASD is conceptualized in other countries and regions. Readers unfamiliar with FASD research in Canada will also benefit from the discussion of CanFASD and NeuroDevNet described as being "comprised of interdisciplinary research teams and are committed to the completion and dissemination of research knowledge to help inform policy and practice and to increase diagnostic, prevention, and intervention efforts of FASD across the country." (Pei et al. 2017, this issue).

Fitzpatrick and Pestell (this issue) describe the advances Australia has made in FASD prevention and intervention in the context of a culture with high rates of alcohol use and engagement of high-risk communities. Indeed, one of the strengths of this article is that it highlights the differences that exist within a country or region in regard to prevalence and attitudes towards alcohol use. Fitzpatrick and Pestell (this issue) note "Rather than taking a deficit-approach through a focus on populations at high risk for FASD in Australia, it is in fact these communities (e.g. Aboriginal and Torres Strait Islander, foster carers) and sectors (Justice, Child protection) 
that have led the call for understanding and action relating to FASD in Australia. For this, mainstream Australian society, where most cases of FASD will reside, can be thankful." (p. 2). Of particular interest to pediatric neuropsychologist who works in remote communities is Fitzpatrick and Pestell's description of the Marulu FASD Prevention Strategy.

As Wang and D'Amato (this issue) point out, despite China being the most populous country in the world, there is a relative lack of conceptualization of FASD by the government, schools, and health care providers. They write "Regarding FASD, there are unmet needs in school, government, mental health services, and the medical community. For example, there is no special education in school emphasizing the detrimental consequences of FASD, no detailed government guidelines on FASD, and no specific health and medical services treating individuals with FASD. FASD does not appear to be a general concern and thus is not widely discussed in any social or medical arena" (Wang \& D'Amato 2017, this issue). Despite these concerns, there are some prevention and intervention elements the authors discuss that are unique to China and this section will be of high interest to readers who want to learn about approaches or effects that are unlikely to be found elsewhere. This includes social pressure, a One-Child policy, mandatory premarital examinations, and premarital education. Overall, an important component of the article is how culture interacts with the conceptualization of FASD in China.

Similar to many of the countries discussed in this Special Issue, alcohol consumption in the UK remains relatively high. As reported by Mukherjee, Carlisle, and Livesy (this issue), a recent study by Nykjaer et al. (2014) indicated $48 \%$ of women consumed alcohol while pregnant and $27 \%$ of women had two units of alcohol a day while pregnant. This relatively high rate of alcohol consumption is a concern given, as Mukherjee et al. point out, that the prevalence of FASD in the UK is largely unknown and to date, there have been no active ascertainment studies conducted in the UK. Furthermore, Mukherjee also report research (Mukherjee et al. (2012) as cited by Mukherjee et al. this issue) that showed people often estimate a standard unit of alcohol to be greater than it is (i.e., pour too much), which confers increased risk for binge drinking. The authors point out an important change that occurred recently in the UK when the guidance changed from one to two alcohol units a week to a recommendation towards abstinence during pregnancy. Readers may also be interested in the discussion of the UK FASD National Clinic including the list of assessments used; this will lend some insight into how clinical practice is conceptualized in the UK.

The inclusion of South Africa (ZA) in this Special Issue is critical as Kalberg, Marais, De Vries, Seedat, Parry, and May (this issue) point out that this country has some of the highest reported prevalence of FASD in the world. The authors describe how collaboration with the USA helped launch epidemiological research on FAS in South Africa. They also describe an interesting approach in which a similar battery of tests is administered during each wave of epidemiological studies, which allows for comparisons over time. In addition to summarizing prevention and intervention efforts, Kalberg et al. discuss the needed role pediatric neuropsychologists and school psychologists play in South Africa for helping to understand FASD and work with affected individuals. The article concludes with a quote which is relevant for all of the countries covered in this Special Issue. "In ZA there is, therefore, a strong need for more pediatric neuropsychologists and school psychologists with an understanding of FASD. It would be ideal if these specifically trained professionals could work hand-in-hand with educational, mental health and public health entities that are also working with these families and individuals to assist individuals to become productive members of society." (Kalberg et al. 2017, this issue).

The final article in this Special Issue looks at a country that, despite a relatively later start in addressing FASD, has been making strides over the past two decades. Okulicz-Kozaryn, Terlikowska, Brzózka, and Borkowska (this issue) note that in the mid-1990s, translations of English-language publications about FASD started to appear in Poland and this grew to a countrywide education program about the effects of prenatal alcohol exposure and subsequent efforts to develop prevention and intervention services for women and children. In this article, Okulicz-Kozaryn et al. report the results of their review of research, positions, and prevention and intervention activities in Poland that have been conducted since the mid-1990s. They review several prevalence studies regarding alcoholexposed pregnancies in Poland and note that other than FAS, other FASD conditions are not recognized in Poland. They conclude that FAS is likely underdiagnosed in Poland and note this is likely a similar concern in other countries. The authors review each of the FASD treatment centers in Poland in some detail including assessment, education, and intervention practices. Similar to the other countries discussed in this Special Issue, there are barriers to the effective implementation of these programs in Poland. Okulicz-Kozaryn notes some concerns including limited research conducted in Poland, unpublished studies, under preparedness of the health care system, and a lack of coordination among FASD treatment centers. They do offer some optimism for continued growth in FASD prevention and intervention including increased involvement and engagement of Polish professional groups and the medical community.

\section{Themes and Future Directions Identified Across Countries}

The articles within this Special Issue highlight several notable themes that have contributed to relative growth in FASD awareness and prevention and intervention efforts within each 
country. Multiple articles referenced the important role of families and community-based advocates in leading the call for increased awareness, policy changes, and responsive services. The establishment of governmental task forces or research networks appears to be a key contributor to larger advances in FASD prevention and intervention. For example, the USA and Canada have well-established, federally and regionally funded research infrastructure that has supported independent and collaborative studies on FASD prevention and intervention. These two countries also have some of the most extensive prevention, diagnostic, and intervention services available, which facilitates such research. Notably, funding has recently been established in Australia to create a National FASD Clinical Network to expand and standardize diagnostic services and facilitate multi-site collaborative clinical research. Other countries, such as Poland and South Africa, have benefitted from the establishment of governmental task forces or agencies to promote FASD awareness and prevention and intervention activities. However, funding for research in these contexts tends to be more sporadic and reliant on other sources. With insufficient formal research infrastructure or governmental support, FASD prevention and intervention efforts rely on dedicated stakeholders, such as family members, clinicians, researchers, and local policy makers, to enact change. Efforts are likely to have the biggest impact when all of these stakeholders work together to achieve a strategic goal.

The contributions to this Special Issue also illustrate some common approaches to FASD prevention and intervention. All articles describe universal prevention strategies that aim to increase awareness of the risks of prenatal alcohol exposure and FASD. Approaches that have been used to convey this information include media campaigns, fact sheets or brochures, premarital education, school-based prevention programs, establishment of national guidelines, and bottle warning labels. Several countries have also demonstrated the efficacy of selective (e.g., targeting women of childbearing age who drink alcohol) and indicated (e.g., targeting pregnant women who are or have used alcohol during pregnancy) prevention programs. Interventions for individuals with FASD have largely focused on preschool and school-aged children and have targeted domains such as self-regulation, attention, social skills, and academic skills. Family-based interventions and supports have also been recognized as important in many countries.

Although progress has been made with respect to FASD prevention and intervention across the countries included in this Special Issue, many challenges remain. One major challenge facing all countries to some degree is identification and diagnosis of FASD. Obtaining accurate information about prenatal alcohol exposure can be complicated by maternal underreporting, stigma, unavailability of documentation, or a lack of appropriate assessment. Another complicating factor is that there are multiple diagnostic systems in use across the world, and even in some countries (e.g., the USA). Although all the existing diagnostic systems generally consider the same basic criteria (facial features, growth, central nervous system functioning, prenatal alcohol exposure), they have different cutoffs and use different diagnostic labels for some conditions across the spectrum. These differences in diagnostic/ classification terms can be confusing to families and providers and result in children being identified inconsistently across systems (Coles et al. 2016). Appropriate normative data (e.g., for facial measurements, head circumference) are also not available for many regions of the world or for various racial/ethnic groups. Sensitive neuropsychological tests may also not be available in all countries, and cultural and linguistic differences can complicate interpretation of scores. Finally, the availability of diagnostic services was generally described as insufficient to meet the needs of children and families, even in portions of countries with the most FASD infrastructure, such as the USA or Canada.

Although several countries described some local successes in establishing prevention and intervention programs, most countries lack coordinated services and supports at a national level and many regions within countries have little to no access to FASD-informed care. The lack of services and supports is most notable in more rural or disadvantaged regions across countries. Another complicating factor is that FAS and/ or FASD more broadly are not recognized as disability categories for service eligibility in educational and/or disabilities service systems in several countries (i.e., the USA, Australia, Poland, some regions of Canada).

All countries in this Special Issue identify insufficient awareness and knowledge about FASD as a primary contributing factor that interferes with prevention and reduces the identification, diagnosis, and effective treatment of affected individuals. This results in a workforce that has limited capacity to address the needs of children and families affected by prenatal alcohol exposure. It also limits the number of stakeholders to advocate for supportive public policies and resource provisions.

Some similar themes emerged across countries for critical actions necessary to address the prevention and intervention needs for FASD. Although intervention research has continued at a steady pace since the first studies were published in the mid-2000s, most studies have been conducted by a handful of research groups in North America. Furthermore, most of these studies have focused on the preschool and school-age developmental period. There is a great need to develop and evaluate theory-driven interventions for affected individuals across the lifespan and their families. Olson (2016) outlines important questions that are vital to advancing FASD intervention research. These questions reflect a systematic approach to intervention research and fall within the following domains: (1) defining the scope of the problem at different developmental stages; (2) defining an efficacious response to the problem; (3) ensuring interventions become evidence- 
based practices; (4) ensuring translation to the community and improving implementation, quality, and utilization; and (5) adapting and creating service systems across the lifespan. As evidenced by the prevention and intervention activities described by individual countries in this Special Issue, additional supports and services are provided to affected individuals and their families in community settings that have not undergone study. Efforts to evaluate these existing programs are recommended.

Research funding and infrastructure are major barriers to prevention and intervention research. Greater advocacy at local, regional, national, and international levels is needed to increase recognition of FASD and the need for research funding for this important and prevalent public health problem. Another related challenge is the limited number of researchers studying prevention and intervention strategies for FASD. Establishing or improving training opportunities for students and new investigators in the field could increase the research workforce. Prevention and intervention research can be challenging and intensive. National and international research networks may be helpful to provide support and exchange of ideas. Opportunities for consultation and coordination across countries could also facilitate establishment of prevention and intervention research in countries with less established infrastructure. For example, the WHO initiated an international project in coordination with the National Institute of Alcohol Abuse and Alcoholism (NIAAA) in the USA to obtain estimates of FASD prevalence in lower- and middle-income countries in Eastern Europe and sub-Saharan Africa. The WHO and NIAAA provided countries with training in a standard study protocol, materials, assistance with funding, and consultation in study implementation within their country. A similar approach could be considered with respect to prevention and intervention through collaborative partnerships and consultation. Understanding local needs and barriers would be essential in developing or adapting interventions for specific regions or countries.

Another theme reiterated across countries is the need for coordinated and integrated supports and services. Even within countries with relatively well-established infrastructure for FASD diagnosis and intervention, services and supports tend to be fragmented across systems (e.g., medical, mental health, education, justice, welfare). Poor coordination and fragmentation of services can be very stressful and frustrating for families as they try to identify services for their child and navigate systems of care. Key issues to be resolved to address this challenge include policies and procedures for cross-system coordination, more training for educators and health care providers, infrastructure changes, and funding. Effective approaches for training a knowledgeable and skilled workforce will also need to be considered. As articulated by Okulicz-Kozaryn, Terlikowska, Brzózka, and Borkowska (this issue), what seems unrealistic at the central level can be more easily achieved at the local level.

\section{The Role of Pediatric Neuropsychologists in FASD Prevention and Intervention}

Pediatric neuropsychologists and psychologists have a valuable role to play in the prevention of and intervention for children with FASD. Pediatric neuropsychologists are important members of the diagnostic team for FASD, as documentation of central nervous system impairment is required for all diagnoses on the spectrum. The profile of neurocognitive strengths and weaknesses uncovered by a pediatric neuropsychologist for each child should inform the development of effective strength-based interventions to address children's functional impairments and improve their adaptive functioning. Additionally, pediatric neuropsychologists are ideally suited to provide psychoeducation to medical professionals, the community, and to women at increased risk of having a child with FASD. This condition is of special concern to psychologists who work with children not only because of the array of life-long neurodevelopmental deficits in cognitive, academic, social, and behavioral functioning but also because it is one of the most preventable causes of intellectual disability.

Children with FASD often present with complex needs and require appropriate interventions to help them function more adaptively across settings. The availability and types of interventions available to children with FASD vary by country and regions within countries. When treatment is available, it is most likely to take place in hospitals, private clinics, homes, or schools. In countries or regions that have well-developed special education systems, schools can be an optimal setting to treat children with this FASD since children spend many of their awake hours in this setting and opportunities for generalization and peer interaction are available. Interventions are likely to be most effective when coordinated across settings. For example, effective communication between the child's caregivers, teachers, and other providers allow interventions to be coordinated across the child's day.

From an intervention perspective, it is imperative to understand each child's neuropsychological profile and what unique deficits are interfering with their functioning in each setting. There is not a specific neuropsychological profile that is associated with FASD, although there are some general commonalities often found across children. Thus, this condition does not result in a homogenous group of children. As such, neuropsychological and educational assessment are critical to ensure children with FASD are receiving strength-based interventions to compensate or remediate their specific weakness(es). Pediatric neuropsychologists have the training and expertise to conduct these types of assessments and consult with educational and mental health professionals to ensure interventions are successfully implemented. 


\section{Conclusion}

Prenatal alcohol exposure is a worldwide public health problem. This Special Issue draws attention to the growth in FASD prevention and intervention efforts across seven countries over the last decade, but also identifies significant challenges worldwide that hinder advances. Despite substantial improvements in educational awareness of the effects of prenatal alcohol exposure, the prevalence of alcohol-exposed pregnancies revealed by the articles in this Special Issue remains unacceptably high. An international perspective of FASD is warranted, especially given the varying beliefs and practices of different cultures regarding alcohol consumption. Additionally, there are synergistic risk factors associated with prenatal alcohol exposure (e.g., poverty, drug use, prenatal care) that vary from country to country. The function and responsibility of psychologists also diverges from one country to another as well as how they provide intervention services. Another difference among cultures and countries is how schools in general, and special education in particular, help children with FASD maximize their potential. Pediatric neuropsychologists and school psychologists in one country or region likely can learn how to better improve their approach by considering what is successful in other countries and regions.

\section{Compliance with Ethical Standards}

Conflict of Interest The authors declare that they have no conflict of interest.

Human and Animal Rights and Informed Consent This article does not contain any studies with human participants or animals performed by the authors.

\section{References}

Coles, C. D., Gailey, A. R., Mulle, J. G., Kable, J. A., Lynch, M. E., \& Jones, K. L. (2016). A comparison among 5 methods for the clinical diagnosis of fetal alcohol spectrum disorders. Alcoholism: Clinical \& Experimental Research, 40, 1000-1009.

Cook, J. L., Green, C. R., Lilley, C. M., Anderson, S. M., Baldwin, M. E., Chudley, A. E., ... Rosales, T. (2016). Fetal alcohol spectrum disorder: a guideline for diagnosis across the lifespan. Canadian Medical Association Journal, 188(3), 191-197.
Kalberg, W. O., Marais, A.-S., De Vries, M. M., Seedat, S., Parry, C., \& May, P. A. (2017). Neuropsychological aspects of prevention and intervention for FASD in South Africa. doi:10.1007/s40817-0170035-2 (in this issue).

May, P. A., Gossage, J. P., Kalberg, W. O., Robinson, L. K., Buckley, D., Manning, M., \& Hoyme, H. E. (2009). Prevalence and epidemiologic characteristics of FASD from various research methods with an emphasis on recent in-school studies. Developmental Disabilities Research Reviews, 15, 176-192.

May, P. A., Blankenship, J., Marais, A., Gossage, J. P., Kalberg, W. O., Barnard, R., De Vries, M., Robinson, L. K., Adnams, C. M., Buckley, D., Manning, M., Jones, K. L., Parry, C., Hoyme, H. E., \& Seedat, S. (2013). Approaching the prevalence of the full spectrum of fetal alcohol spectrum disorders in a South African population-based study. Alcoholism: Clinical and Experimental Research, 37, 818-830.

Mukherjee, R. A. S., Wray, E., Curfs, L., \& Hollins, S. (2012). Estimation of alcohol content of wine, beer and spirits to evaluate exposure risk in pregnancy: pilot study using a questionnaire and pouring task in England. International Journal of Alcohol and Drugs.

Nykjaer, C., Alwan, N. A., Greenwood, D. C., Simpson, N. A. B., Hay, A. W. M., White, K. L. M., et al. (2014). Maternal alcohol intake prior to and during pregnancy and risk of adverse birth outcomes: evidence from a British cohort. Journal Epidemiology and Community Health, 68, 542-549.

Olson, H. C. (2016). A renewed call to action: the need for systematic research on interventions for FASD. Alcoholism: Clinical and Experimental Research, 40, 1817-1821.

Pei, J., Tremblay, M., McNeil, A., Poole, N., \& McFarlane, A. (2017). Neuropsychological aspects of prevention and intervention for FASD in Canada. doi:10.1007/s40817-016-0020-1 (in this issue).

Popova, S., Stade, B., Bekmuradov, Lange, S., \& Rehm, J. (2011). What do we know about the economic impact of fetal alcohol spectrum disorder? A literature review. Alcohol and Alcoholism, 46, 490-497.

Popova, S., Lange, S., Probst, C., Gmel, G., \& Rehm, J. (2017). Estimation of national, regional, and global prevalence of alcohol use during pregnancy and fetal alcohol syndrome: a systematic review and meta-analysis. Lancet Global Health. Published online January 12, 2017. doi:10.1016/S2214-109X(17)30021-9.

Streissguth, A. P., Bookstein, F. L., Barr, H. M., Sampson, P. D., O'Malley, K., \& Young, J. K. (2004). Risk factors for adverse life outcomes in fetal alcohol syndrome and fetal alcohol effects. Journal of Developmental and Behavioral Pediatrics, 25, 228-238.

Wang, Y. Y., D'Amato, R. C. (2017). Understanding fetal alcohol spectrum disorders in China. doi:10.1007/s40817-017-0029-0 (in this issue).

World Health Organization (2005). Alcohol, gender, and drinking problems: perspectives from low- and middle-income countries. Geneva.

World Health Organization (2015). Global status report on alcohol and health. Geneva. 